ANNALES

POLONICI MATHEMATICI

$95.3(2009)$

\title{
Liftings of forms to Weil bundles and the exterior derivative
}

\author{
by JACEK DęBECKI (Kraków)
}

\begin{abstract}
In a previous paper we have given a complete description of linear liftings of $p$-forms on $n$-dimensional manifolds $M$ to $q$-forms on $T^{A} M$, where $T^{A}$ is a Weil functor, for all non-negative integers $n, p$ and $q$, except the case $p=n$ and $q=0$. We now establish formulas connecting such liftings and the exterior derivative of forms. These formulas contain a boundary operator, which enables us to define a homology of the Weil algebra $A$. We next study the case $p=n$ and $q=0$ under the condition that $A$ is acyclic. Finally, we compute the kernels and the images of the boundary operators for the Weil algebras $\mathbb{D}_{k}^{r}$ and show that these algebras are acyclic.
\end{abstract}

Linear liftings of forms to Weil bundles. There is a well known oneto-one correspondence between product preserving bundle functors, which are also called Weil functors or Weil bundles, and Weil algebras ([4], [7], [10]). Consequently, one may try to describe some natural operators transforming sections of a natural bundle into sections of another natural bundle over a Weil bundle (we call natural operators of this kind simply liftings) in terms of the corresponding Weil algebra (see [9] for the general theory of natural operators). In particular, liftings of forms to Weil bundles have been studied by several authors (see for instance [5], [6], [11], [12], [3], [1]).

In this section we briefly recall basic definitions and results of [1], as they are the starting point of the present paper.

We will denote by $F_{p} M$ the vector space of $p$-forms on a manifold $M$. Let $A$ be a Weil algebra and $T^{A}$ the Weil functor corresponding to $A$ (see [9]). Fix non-negative integers $n, p$ and $q$. A linear lifting of $p$-forms to $q$-forms on $T^{A}$ is, by definition, a system of linear maps $L_{M}: F_{p} M \rightarrow F_{q}\left(T^{A} M\right)$ indexed by $n$-dimensional manifolds and satisfying for all such manifolds $M$ and $N$, every embedding $f: M \rightarrow N$ and every $\omega \in F_{p} N$ the condition

$$
L_{M}\left(f^{*} \omega\right)=\left(T^{A} f\right)^{*}\left(L_{N}(\omega)\right) .
$$

2000 Mathematics Subject Classification: Primary 58A32.

Key words and phrases: natural operator, Weil bundle. 
If $s$ is a non-negative integer, then we will use the symbol $C_{s} A$ to denote the vector space of skew-symmetric s-linear maps $G: A \times \cdots \times A \rightarrow A^{*}$ (where $A^{*}$ denotes the vector space of linear functions $A \rightarrow \mathbb{R}$ ) satisfying

$$
G\left(a_{1}, \ldots, a_{s-1}, b c\right)(d)=G\left(a_{1}, \ldots, a_{s-1}, b\right)(c d)+G\left(a_{1}, \ldots, a_{s-1}, c\right)(b d)
$$

for all $a_{1}, \ldots, a_{s-1}, b, c, d \in A$ whenever $s \geq 1$.

Suppose $p \geq q$ and $D \in C_{p-q} A$. There is a unique linear lifting $D^{p, q}$ of $p$-forms to $q$-forms on $T^{A}$ such that

$$
\begin{aligned}
D_{U}^{p, q}(\omega)(X)\left(Y_{1}, \ldots, Y_{q}\right) & \\
& =D\left(X^{i_{q+1}}, \ldots, X^{i_{p}}\right)\left(\left(\left(T^{A} \omega_{i_{1} \ldots i_{p}}\right)(X)\right) Y_{1}^{i_{1}} \ldots Y_{q}^{i_{q}}\right)
\end{aligned}
$$

for every open subset $U$ of $\mathbb{R}^{n}$, every $\omega \in F_{p} U$, every $X \in T^{A} U$ and all $Y_{1}, \ldots, Y_{q} \in A^{n}$, where the coordinates $\omega_{i_{1} \ldots i_{p}}: U \rightarrow \mathbb{R}$ for $i_{1}, \ldots, i_{p} \in$ $\{1, \ldots, n\}$ are such that $\omega(x)\left(y_{1}, \ldots, y_{p}\right)=\omega_{i_{1} \ldots i_{p}}(x) y_{1}^{i_{1}} \ldots y_{p}^{i_{p}}$ for every $x \in U$ and all $y_{1}, \ldots, y_{p} \in \mathbb{R}^{n}$ (formula (3) makes sense, because $T^{A} \mathbb{R}^{n}=A^{n}$ and $T^{A} U$ is an open subset of the vector space $A^{n}$, so $D_{U}^{p, q}(\omega)(X)$ can be interpreted as a skew-symmetric $q$-linear map $\left.A^{n} \times \cdots \times A^{n} \rightarrow \mathbb{R}\right)$.

If $p+1 \geq q$ and $E \in C_{p-q+1} A$, then $E^{p+1, q} \circ d$ (where $d$ denotes the exterior derivative) is another linear lifting of $p$-forms to $q$-forms on $T^{A}$. The main theorem of [1] states that every linear lifting of $p$-forms to $q$-forms on $T^{A}$ is of the form specified in the table below for almost all $n, p$ and $q$, where $D \in C_{p-q} A$ and $E \in C_{p-q+1} A$ are uniquely determined.

\begin{tabular}{cccc}
\hline & $0 \leq p \leq n-1$ & $p=n$ & $n+1 \leq p$ \\
\hline$q=0$ & $D^{p, 0}$ & 0 \\
$1 \leq q \leq p$ & $D^{p, q}+E^{p+1, q} \circ d$ & $D^{n, q}$ & 0 \\
$q=p+1$ & $E^{p+1, p+1} \circ d$ & 0 & 0 \\
$p+2 \leq q$ & 0 & 0 & 0 \\
\hline
\end{tabular}

The case $p=n$ and $q=0$ was omitted in [1], because it is more difficult than the others. In what follows we prove Theorem 2, which covers this unusual case.

A homology of the algebra $A$. If $p \geq q-1 \geq 0$ and $F \in C_{p-q+1} A$ then $d \circ F^{p, q-1}$ is a linear lifting of $p$-forms to $q$-forms on $T^{A}$, and so it is of the form specified in the above table with some $D$ and $E$ determined by $F$. In the next section we will establish explicit formulas for $d \circ F^{p, q-1}$. They will contain a boundary operator, which we now define. 
For every positive integer $s$ and every $G \in C_{s} A$ we define the skewsymmetric $(s-1)$-linear map $\partial_{s} G: A \times \cdots \times A \rightarrow A^{*}$ by

$$
\left(\partial_{s} G\right)\left(a_{1}, \ldots, a_{s-1}\right)(b)=G\left(a_{1}, \ldots, a_{s-1}, b\right)(1)
$$

for all $a_{1}, \ldots, a_{s-1}, b \in A$. We will have $\partial_{s} G \in C_{s-1} A$ once we check that

$$
\begin{aligned}
\left(\partial_{s} G\right)\left(a_{1}, \ldots, a_{s-2}, b c\right)(d) & \\
& =\left(\partial_{s} G\right)\left(a_{1}, \ldots, a_{s-2}, b\right)(c d)+\left(\partial_{s} G\right)\left(a_{1}, \ldots, a_{s-2}, c\right)(b d)
\end{aligned}
$$

for all $a_{1}, \ldots, a_{s-2}, b, c, d \in A$ whenever $s \geq 2$. By (2) and the skew-symmetry of $G$,

$$
\begin{aligned}
&\left(\partial_{s} G\right)\left(a_{1}, \ldots, a_{s-2}, b c\right)(d)=G\left(a_{1}, \ldots, a_{s-2}, b c, d\right)(1) \\
&= G\left(a_{1}, \ldots, a_{s-2}, b, d\right)(c)+G\left(a_{1}, \ldots, a_{s-2}, c, d\right)(b) \\
&= G\left(a_{1}, \ldots, a_{s-2}, b, c\right)(d)+G\left(a_{1}, \ldots, a_{s-2}, b, d\right)(c) \\
&+G\left(a_{1}, \ldots, a_{s-2}, c, b\right)(d)+G\left(a_{1}, \ldots, a_{s-2}, c, d\right)(b) \\
&= G\left(a_{1}, \ldots, a_{s-2}, b, c d\right)(1)+G\left(a_{1}, \ldots, a_{s-2}, c, b d\right)(1) \\
&=\left(\partial_{s} G\right)\left(a_{1}, \ldots, a_{s-2}, b\right)(c d)+\left(\partial_{s} G\right)\left(a_{1}, \ldots, a_{s-2}, c\right)(b d),
\end{aligned}
$$

which is the desired conclusion. Therefore we have defined the linear map $\partial_{s}: C_{s} A \rightarrow C_{s-1} A$ for every positive integer $s$.

It will be convenient to put $C_{-1} A=\mathbb{R}, \partial_{0}: C_{0} A \ni G \mapsto G(1) \in C_{-1} A$ (this definition makes sense, because $C_{0} A=A^{*}$ ) and $\partial_{-1}=0$.

If $s \geq 1$ and $G \in C_{s} A$, then by (2),

$$
\begin{aligned}
G\left(a_{1}, \ldots, a_{s-1}, 1\right)(1) & =G\left(a_{1}, \ldots, a_{s-1}, 1 \cdot 1\right)(1) \\
& =G\left(a_{1}, \ldots, a_{s-1}, 1\right)(1)+G\left(a_{1}, \ldots, a_{s-1}, 1\right)(1),
\end{aligned}
$$

and so $G\left(a_{1}, \ldots, a_{s-1}, 1\right)(1)=0$ for all $a_{1}, \ldots, a_{s-1} \in A$. From this we deduce that $\partial_{s-1} \partial_{s}=0$ for every $s \geq 0$.

Thus we have constructed a chain complex and we define the homology vector spaces of the algebra $A$ by the usual formula $H_{s} A=\operatorname{ker} \partial_{s} / \operatorname{im} \partial_{s+1}$ for every $s \geq-1$. We will call $A$ acyclic if $H_{s} A=\{0\}$ for all $s \geq-1$.

As a curiosity, it is worth pointing out that the above construction works not only for a Weil algebra, but also for any algebra with unit, even noncommutative and non-associative.

Formulas for $d \circ F^{p, q-1}$. Since constant coefficients in formulas we want to derive depend on the choice of constant coefficients in some definitions, we now make the following convention. If $m$ and $s$ are non-negative integers, $V$ is an open subset of $\mathbb{R}^{m}, \alpha \in F_{s} V$ and the coordinates $\alpha_{i_{1} \ldots i_{s}}: V \rightarrow \mathbb{R}$ for $i_{1}, \ldots, i_{s} \in\{1, \ldots, m\}$ are such that $\alpha(x)\left(y_{1}, \ldots, y_{s}\right)=\alpha_{i_{1} \ldots i_{s}}(x) y_{1}^{i_{1}} \ldots y_{s}^{i_{s}}$ 
for every $x \in V$ and all $y_{1}, \ldots, y_{s} \in \mathbb{R}^{m}$, then $\alpha=\alpha_{i_{1} \ldots i_{s}} d x^{i_{1}} \wedge \cdots \wedge d x^{i_{s}}$ and

$$
d \alpha=\frac{\partial \alpha_{i_{1} \ldots i_{s}}}{\partial x^{j}} d x^{j} \wedge d x^{i_{1}} \wedge \cdots \wedge d x^{i_{s}} .
$$

We are now in a position to formulate a theorem containing the promised formulas for $d \circ F^{p, q-1}$, where $F \in C_{p-q+1} A$.

Theorem 1. Let $p-q+1 \geq 0$ and $F \in C_{p-q+1} A$.

If $q=0$, then

$$
0=(-1)^{p-1}\left(\partial_{p+1} F\right)^{p, 0}+F^{p+1,0} \circ d .
$$

If $1 \leq q \leq p$, then

$$
q\left(d \circ F^{p, q-1}\right)=(p-q+1)(-1)^{p-1}\left(\partial_{p-q+1} F\right)^{p, q}+(p+1)\left(F^{p+1, q} \circ d\right) .
$$

If $q=p+1$, then

$$
d \circ F^{p, p}=F^{p+1, p+1} \circ d .
$$

Proof. To simplify the proof, note that (4) and (6) may be interpreted as special cases of (5), because the coefficients of the terms which do not make sense vanish in these special cases.

If $s$ is a positive integer, $u$ is a non-negative integer and $G \in C_{s} A$, then by induction on $u$ we deduce from (2) that

$$
\begin{aligned}
G\left(a_{1}, \ldots, a_{s-1}, b_{1} \ldots b_{u}\right)(c) & \\
& =\sum_{v=1}^{u} G\left(a_{1}, \ldots, a_{s-1}, b_{v}\right)\left(b_{1} \ldots b_{v-1} b_{v+1} \ldots b_{u} c\right)
\end{aligned}
$$

for all $a_{1}, \ldots, a_{s-1}, b_{1}, \ldots, b_{u}, c \in A$. From (7) we conclude immediately that if $f: \mathbb{R}^{n} \rightarrow \mathbb{R}$ is a polynomial, then

$$
\begin{aligned}
& G\left(a_{1}, \ldots, a_{s-1},\left(T^{A} f\right)\right.(X))(c) \\
&=G\left(a_{1}, \ldots, a_{s-1}, X^{j}\right)\left(\left(\left(T^{A} \frac{\partial f}{\partial x^{j}}\right)(X)\right) c\right)
\end{aligned}
$$

for every $X \in A^{n}$. Actually, (8) still holds when $f: U \rightarrow \mathbb{R}$ is a smooth map on an open subset $U$ of $\mathbb{R}^{n}$ and $X \in T^{A} U$ (this is due to the fact that $T^{A}$ has a finite order; see [1]).

If $q \geq 1, m$ is a non-negative integer, $V$ is an open subset of $\mathbb{R}^{m}$ and $\alpha \in F_{q-1} V$, then we write $\alpha^{y_{1} \ldots y_{q-1}}: V \ni x \mapsto \alpha(x)\left(y_{1}, \ldots, y_{q-1}\right) \in \mathbb{R}$ for $y_{1}, \ldots, y_{q-1} \in \mathbb{R}^{m}$. In this notation we have

$$
(d \alpha)(x)\left(y_{1}, \ldots, y_{q}\right)=\frac{1}{q} \sum_{u=1}^{q}(-1)^{u-1}\left(d\left(\alpha^{y_{1} \ldots y_{u-1} y_{u+1} \ldots y_{q}}\right)\right)(x)\left(y_{u}\right)
$$

for every $x \in V$ and all $y_{1}, \ldots, y_{q} \in \mathbb{R}^{m}$, as is easy to verify. 
Let $U$ be an open subset of $\mathbb{R}^{n}$ and $\omega \in F_{p} U$. By (3), if $q \geq 1$ then

$$
\begin{aligned}
F_{U}^{p, q-1}(\omega)(X)\left(Y_{1}, \ldots, Y_{q-1}\right) & \\
& =F\left(X^{i_{q}}, \ldots, X^{i_{p}}\right)\left(\left(\left(T^{A} \omega_{i_{1} \ldots i_{p}}\right)(X)\right) Y_{1}^{i_{1}} \ldots Y_{q-1}^{i_{q-1}}\right)
\end{aligned}
$$

for every $X \in T^{A} U$ and all $Y_{1}, \ldots, Y_{q-1} \in A^{n}$. Hence (9) gives

$$
\begin{gathered}
=\sum_{u=1}^{q}(-1)^{u-1}\left(\sum_{t=q}^{p} F\left(X^{i_{q}}, \ldots, X^{i_{t-1}}, Y_{u}^{i_{t}}, X^{i_{t+1}}, \ldots, X^{i_{p}}\right)\right. \\
\left(\left(\left(T^{A} \omega_{i_{1} \ldots i_{p}}\right)(X)\right) Y_{1}^{i_{1}} \ldots Y_{u-1}^{i_{u-1}} Y_{u+1}^{i_{u}} \ldots Y_{q}^{i_{q-1}}\right) \\
\left.+F\left(X^{i_{q}}, \ldots, X^{i_{p}}\right)\left(\left(\left(T^{A} \frac{\partial \omega_{i_{1} \ldots i_{p}}}{\partial x^{j}}\right)(X)\right) Y_{1}^{i_{1}} \ldots Y_{u-1}^{i_{u-1}} Y_{u+1}^{i_{u}} \ldots Y_{q}^{i_{q-1}} Y_{u}^{j}\right)\right)
\end{gathered}
$$

for every $X \in T^{A} U$ and all $Y_{1}, \ldots, Y_{q} \in A^{n}$. But, by the skew-symmetry of $F$ and $\omega$,

$$
\begin{aligned}
\text { (11) } \sum_{u=1}^{q}(-1)^{u-1} \sum_{t=q}^{p} F\left(X^{i_{q}}, \ldots, X^{i_{t-1}}, Y_{u}^{i_{t}}, X^{i_{t+1}}, \ldots, X^{i_{p}}\right) & \\
& \left(\left(\left(T^{A} \omega_{i_{1} \ldots i_{p}}\right)(X)\right) Y_{1}^{i_{1}} \ldots Y_{u-1}^{i_{u-1}} Y_{u+1}^{i_{u}} \ldots Y_{q}^{i_{q-1}}\right) \\
= & (p-q+1) \sum_{u=1}^{q}(-1)^{u-1}
\end{aligned}
$$$$
\times F\left(X^{i_{q}}, \ldots, X^{i_{p-1}}, Y_{u}^{i_{p}}\right)\left(\left(\left(T^{A} \omega_{i_{1} \ldots i_{p}}\right)(X)\right) Y_{1}^{i_{1}} \ldots Y_{u-1}^{i_{u-1}} Y_{u+1}^{i_{u}} \ldots Y_{q}^{i_{q-1}}\right)
$$$$
=(p-q+1)(-1)^{p-1}
$$$$
\times \sum_{u=1}^{q} F\left(X^{i_{q+1}}, \ldots, X^{i_{p}}, Y_{u}^{i_{u}}\right)\left(\left(\left(T^{A} \omega_{i_{1} \ldots i_{p}}\right)(X)\right) Y_{1}^{i_{1}} \ldots Y_{u-1}^{i_{u-1}} Y_{u+1}^{i_{u+1}} \ldots Y_{q}^{i_{q}}\right) .
$$

Since

$$
(d \omega)_{i_{1} \ldots i_{p+1}}=\frac{1}{p+1} \sum_{u=1}^{p+1}(-1)^{u-1} \frac{\partial \omega_{i_{1} \ldots i_{u-1} i_{u+1} \ldots i_{p+1}}}{\partial x^{i_{u}}}
$$

for all $i_{1}, \ldots, i_{p+1} \in\{1, \ldots, n\},(3)$ and the skew-symmetry of $F$ give

$$
(p+1)\left(F_{U}^{p+1, q}(d \omega)\right)(X)\left(Y_{1}, \ldots, Y_{q}\right)
$$

$$
=\sum_{u=1}^{p+1}(-1)^{u-1} F\left(X^{i_{q+1}}, \ldots, X^{i_{p+1}}\right)\left(\left(\left(T^{A} \frac{\partial \omega_{i_{1} \ldots i_{u-1} i_{u+1} \ldots i_{p+1}}}{\partial x^{i_{u}}}\right)(X)\right) Y_{1}^{i_{1}} \ldots Y_{q}^{i_{q}}\right)
$$




$$
\begin{aligned}
& =\sum_{u=1}^{q}(-1)^{u-1} F\left(X^{i_{q}}, \ldots, X^{i_{p}}\right)\left(\left(\left(T^{A} \frac{\partial \omega_{i_{1} \ldots i_{p}}}{\partial x^{j}}\right)(X)\right) Y_{1}^{i_{1}} \ldots Y_{u-1}^{i_{u-1}} Y_{u+1}^{i_{u}} \ldots Y_{q}^{i_{q-1}} Y_{u}^{j}\right) \\
& =(-1)^{p} \sum_{u=q+1}^{p+1} F\left(X^{i_{q+1}}, \ldots, X^{i_{p}}, X^{j}\right)\left(\left(\left(T^{A} \frac{\partial \omega_{i_{1} \ldots i_{p}}}{\partial x^{j}}\right)(X)\right) Y_{1}^{i_{1}} \ldots Y_{q}^{i_{q}}\right)
\end{aligned}
$$

for every $X \in T^{A} U$ and all $Y_{1}, \ldots, Y_{q} \in A^{n}$. But, by (8),

$$
\begin{gathered}
(-1)^{p} \sum_{u=q+1}^{p+1} F\left(X^{i_{q+1}}, \ldots, X^{i_{p}}, X^{j}\right)\left(\left(\left(T^{A} \frac{\partial \omega_{i_{1} \ldots i_{p}}}{\partial x^{j}}\right)(X)\right) Y_{1}^{i_{1}} \ldots Y_{q}^{i_{q}}\right) \\
+(p-q+1)(-1)^{p} F\left(X^{i_{q+1}}, \ldots, X^{i_{p}},\left(T^{A} \omega_{i_{1} \ldots i_{p}}\right)(X)\right)\left(Y_{1}^{i_{1}} \ldots Y_{q}^{i_{q}}\right) .
\end{gathered}
$$

Combining (10)-(13) and applying (7) and (3) we obtain

$$
\begin{aligned}
& \left(q\left(d\left(F_{U}^{p, q-1}(\omega)\right)\right)-(p+1)\left(F_{U}^{p+1, q}(d \omega)\right)\right)(X)\left(Y_{1}, \ldots, Y_{q}\right) \\
& =(p-q+1)(-1)^{p-1} \\
& \quad \times \sum_{u=1}^{q} F\left(X^{i_{q+1}}, \ldots, X^{i_{p}}, Y_{u}^{i_{u}}\right)\left(\left(\left(T^{A} \omega_{i_{1} \ldots i_{p}}\right)(X)\right) Y_{1}^{i_{1}} \ldots Y_{u-1}^{i_{u-1}} Y_{u+1}^{i_{u+1}} \ldots Y_{q}^{i_{q}}\right) \\
& \quad-(p-q+1)(-1)^{p} F\left(X^{i_{q+1}}, \ldots, X^{i_{p}},\left(T^{A} \omega_{i_{1} \ldots i_{p}}\right)(X)\right)\left(Y_{1}^{i_{1}} \ldots Y_{q}^{i_{q}}\right) \\
& =(p-q+1)(-1)^{p-1} F\left(X^{i_{q+1}}, \ldots, X^{i_{p}},\left(\left(T^{A} \omega_{i_{1} \ldots i_{p}}\right)(X)\right) Y_{1}^{i_{1}} \ldots Y_{q}^{i_{q}}\right)(1) \\
& =(p-q+1)(-1)^{p-1}\left(\partial_{p-q+1} F\right)_{U}^{p, q}(\omega)(X)\left(Y_{1}, \ldots, Y_{q}\right)
\end{aligned}
$$

for every $X \in T^{A} U$ and all $Y_{1}, \ldots, Y_{q} \in A^{n}$. This establishes (5), and the proof is complete.

The case $p=n$ and $q=0$. Let $L$ be a linear lifting of $n$-forms to 0 -forms on $T^{A}$. One may ask if there exists a $D \in C_{n} A$ such that $L=D^{n, 0}$ and, if this is so, whether such a $D$ is unique or not. The following proposition answers the latter question.

Proposition 1. Let $D, \widetilde{D} \in C_{n} A$. Then $D^{n, 0}=\widetilde{D}^{n, 0}$ if and only if $\partial_{n} D=\partial_{n} \widetilde{D}$.

Proof. From a lemma proved in [1] it follows that if $L$ and $\widetilde{L}$ are two linear liftings of $n$-forms to 0 -forms on $T^{A}$ such that

$$
L_{\mathbb{R}^{n}}\left(d x^{1} \wedge \cdots \wedge d x^{n}\right)=\widetilde{L}_{\mathbb{R}^{n}}\left(d x^{1} \wedge \cdots \wedge d x^{n}\right),
$$

then $L=\widetilde{L}$. By (3) and the skew-symmetry of $D$,

$$
\begin{aligned}
& D_{\mathbb{R}^{n}}^{n, 0}\left(d x^{1} \wedge \cdots \wedge d x^{n}\right)(X) \\
& =D\left(X^{1}, \ldots, X^{n}\right)(1)= \begin{cases}\left(\partial_{n} D\right)\left(X^{1}, \ldots, X^{n-1}\right)\left(X^{n}\right) & \text { if } n \geq 1, \\
\partial_{0} D & \text { if } n=0,\end{cases}
\end{aligned}
$$


for every $X \in A^{n}$, and a similar formula holds for $\widetilde{D}$. Combining these with the above-mentioned lemma completes the proof.

The problem of the existence of a $D \in C_{n} A$ such that $L=D^{n, 0}$ is more difficult. We will solve it under the condition that $H_{n-1} A=\{0\}$.

We define $S_{n-1} A$ to be the set of skew-symmetric $n$-linear functions $C$ : $A \times \cdots \times A \rightarrow \mathbb{R}$ with the properties that

$$
C\left(a_{1}, \ldots, a_{n-2}, b c, d\right)=C\left(a_{1}, \ldots, a_{n-2}, b, c d\right)+C\left(a_{1}, \ldots, a_{n-2}, c, b d\right)
$$

for all $a_{1}, \ldots, a_{n-2}, b, c, d \in A$ whenever $n \geq 2$ and

$$
C\left(a_{1}, \ldots, a_{n-1}, 1\right)=0
$$

for all $a_{1}, \ldots, a_{n-1} \in A$ whenever $n \geq 1$ (note that if $n \geq 2$, then (16) is a consequence of (15) with $b=1$ and $c=1)$.

Let $C \in S_{n-1} A$. If $n \geq 1$, then we define the skew-symmetric $(n-1)$-linear $\operatorname{map} \bar{C}: A \times \cdots \times A \rightarrow A^{*}$ by

$$
\bar{C}\left(a_{1}, \ldots, a_{n-1}\right)(b)=C\left(a_{1}, \ldots, a_{n-1}, b\right)
$$

for $a_{1}, \ldots, a_{n-1}, b \in A$. If $n=0$, then we put $\bar{C}=C$ (in this case $C \in \mathbb{R}$ ). Let $\overline{S_{n-1} A}$ denote the image of the linear injection $S_{n-1} A \ni C \mapsto \bar{C} \in \operatorname{ker} \partial_{n-1}$.

Proposition 2. $\overline{S_{n-1} A}=\operatorname{ker} \partial_{n-1}$.

Proof. The case $n \leq 1$ is trivial. Suppose that $n \geq 2$ and $G \in \operatorname{ker} \partial_{n-1}$. The $n$-linear function $C: A \times \cdots \times A \rightarrow \mathbb{R}$ given by $C\left(a_{1}, \ldots, a_{n-1}, b\right)=$ $G\left(a_{1}, \ldots, a_{n-1}\right)(b)$ for all $a_{1}, \ldots, a_{n-1}, b \in A$ is skew-symmetric in the first $n-1$ variables. If we show that it is skew-symmetric in the last two variables as well, we will have $G=\bar{C}$ and the proof will be completed. But the fact that $\partial_{n-1} G=0$ and (2) yield

$$
0=G\left(a_{1}, \ldots, a_{n-2}, b c\right)(1)=G\left(a_{1}, \ldots, a_{n-2}, b\right)(c)+G\left(a_{1}, \ldots, a_{n-2}, c\right)(b)
$$

for all $a_{1}, \ldots, a_{n-2}, b, c \in A$, which is the desired conclusion.

LEMMA 1. For every linear lifting $L$ of $n$-forms to 0 -forms on $T^{A}$ there is a unique $C \in S_{n-1} A$ such that

$$
L_{\mathbb{R}^{n}}\left(d x^{1} \wedge \cdots \wedge d x^{n}\right)(X)=C\left(X^{1}, \ldots, X^{n}\right)
$$

for every $X=\left(X^{1}, \ldots, X^{n}\right) \in A^{n}$.

Proof. From (1) with $f: \mathbb{R}^{n} \ni x \mapsto\left(t_{1} x^{1}, \ldots, t_{n} x^{n}\right) \in \mathbb{R}^{n}$, where $t_{1}, \ldots, t_{n} \in \mathbb{R} \backslash\{0\}$, we have

$$
t_{1} \ldots t_{n} L_{\mathbb{R}^{n}}\left(d x^{1} \wedge \cdots \wedge d x^{n}\right)(X)=L_{\mathbb{R}^{n}}\left(d x^{1} \wedge \cdots \wedge d x^{n}\right)\left(t_{1} X^{1}, \ldots, t_{n} X^{n}\right)
$$

for every $X \in A^{n}$. By continuity, the same is true for all $t_{1}, \ldots, t_{n} \in \mathbb{R}$. The homogeneous function theorem (see [9]) now shows that there is an $n$-linear function $C: A \times \cdots \times A \rightarrow \mathbb{R}$ satisfying (17). 
From (1) with $f: \mathbb{R}^{n} \ni x \mapsto\left(x^{\sigma(1)}, \ldots, x^{\sigma(n)}\right) \in \mathbb{R}^{n}$, where $\sigma$ is a permutation of $\{1, \ldots, n\}$, we have

$$
\operatorname{sgn} \sigma L_{\mathbb{R}^{n}}\left(d x^{1} \wedge \cdots \wedge d x^{n}\right)(X)=L_{\mathbb{R}^{n}}\left(d x^{1} \wedge \cdots \wedge d x^{n}\right)\left(X^{\sigma(1)}, \ldots, X^{\sigma(n)}\right)
$$

for every $X \in A^{n}$. Hence, by (17), $C$ is skew-symmetric.

If $n \geq 2$, then put $U=\left\{x \in \mathbb{R}^{n}: x^{n-1}>0\right\}$ and

$$
\begin{aligned}
& f: U \ni x \mapsto\left(x^{1}, \ldots, x^{n-2}, \frac{1}{2}\left(x^{n-1}\right)^{2}, x^{n}\right) \in \mathbb{R}^{n}, \\
& g: U \ni x \mapsto\left(x^{1}, \ldots, x^{n-1}, x^{n-1} x^{n}\right) \in \mathbb{R}^{n} .
\end{aligned}
$$

Since $f^{*}\left(d x^{1} \wedge \cdots \wedge d x^{n}\right)=g^{*}\left(d x^{1} \wedge \cdots \wedge d x^{n}\right)$, from (1) we have

$$
\begin{aligned}
L_{\mathbb{R}^{n}}\left(d x^{1} \wedge \cdots \wedge d x^{n}\right)\left(X^{1}, \ldots, X^{n-2}, \frac{1}{2}\left(X^{n-1}\right)^{2}, X^{n}\right) \\
\quad=L_{\mathbb{R}^{n}}\left(d x^{1} \wedge \cdots \wedge d x^{n}\right)\left(X^{1}, \ldots, X^{n-1}, X^{n-1} X^{n}\right)
\end{aligned}
$$

for $X \in T^{A} U$. In the same manner, with $U$ replaced by $\left\{x \in \mathbb{R}^{n}: x^{n-1}<0\right\}$, we can see that (18) also holds for $X \in T^{A}\left\{x \in \mathbb{R}^{n}: x^{n-1}<0\right\}$, and so, by continuity, for every $X \in A^{n}$. The polarization of (18) with respect to $X^{n-1}$ and (17) now show that (15) holds.

If $n=1$, then from (1) with $f: \mathbb{R} \ni x \mapsto 2 x-1 \in \mathbb{R}$ we have $2 L_{\mathbb{R}}(d x)(1)$ $=L_{\mathbb{R}}(d x)(1)$, so $L_{\mathbb{R}}(d x)(1)=0$. Hence, by (17), $C$ satisfies (16).

The uniqueness of $C$ is evident. This completes the proof.

Lemma 1 enables us to formulate the following proposition.

Proposition 3. Let $L$ be a linear lifting of $n$-forms to 0 -forms on $T^{A}$ and $C \in S_{n-1} A$ be such that (17) holds. Then there exists a $D \in C_{n} A$ such that $L=D^{n, 0}$ if and only if $\bar{C} \in \operatorname{im} \partial_{n}$.

Proof. If $D \in C_{n} A$, then (17) and (14) show that $\bar{C}=\partial_{n} D$ if and only if $L_{\mathbb{R}^{n}}\left(d x^{1} \wedge \cdots \wedge d x^{n}\right)=D_{\mathbb{R}^{n}}^{n, 0}\left(d x^{1} \wedge \cdots \wedge d x^{n}\right)$, which is equivalent to $L=D^{n, 0}$, because of the above-mentioned lemma of [1]. This completes the proof.

Propositions 1-3 give the following theorem.

Theorem 2. If $H_{n-1} A=\{0\}$, then for every linear lifting $L$ of $n$-forms to 0 -forms on $T^{A}$ there is a $D \in C_{n} A$ such that $L=D^{n, 0}$, and so the vector space of linear liftings of $n$-forms to 0 -forms on $T^{A}$ is isomorphic to $C_{n} A / \operatorname{ker} \partial_{n}$.

Thus Theorem 2 gives a classification of linear liftings of $n$-forms to 0 forms on $T^{A}$ under the condition that $A$ is acyclic. Clearly, ker $\partial_{-1}=\mathbb{R}$ and $\operatorname{im} \partial_{0}=\mathbb{R}$. However, computing $\operatorname{ker} \partial_{s}$ and $\operatorname{im} \partial_{s+1}$ for all $s \geq-1$ and an arbitrary Weil algebra $A$ seems difficult. In the next section we will solve this problem for some special (but important) Weil algebras.

$\operatorname{ker} \partial_{s}$ and $\operatorname{im} \partial_{s+1}$ for the algebras $\mathbb{D}_{k}^{r}$. Let $r$ and $k$ be non-negative integers. We will denote by $\mathbb{D}_{k}^{r}$ the Weil algebra of $r$-jets at 0 of smooth 
functions $\mathbb{R}^{k} \rightarrow \mathbb{R}$. It is worth pointing out that each Weil algebra $A$ is a factor algebra of $\mathbb{D}_{k}^{r}$ for some $r$ and $k$ (see [8]), so the chain complex $C A$ is isomorphic to a subcomplex of $C \mathbb{D}_{k}^{r}$.

If $r=0$ or $k=0$, then $\mathbb{D}_{k}^{r}=\mathbb{R}$, and it is a simple matter to check that $H_{s} \mathbb{R}=\{0\}$ for every $s \geq-1$.

From now on we make the assumption that $r \geq 1$ and $k \geq 1$.

We will denote by $x^{i}$ for $i \in\{1, \ldots, k\}$ the $r$-jet at 0 of the projection $\mathbb{R}^{k} \ni x \mapsto x^{i} \in \mathbb{R}$ and write $x^{\alpha}=\left(x^{1}\right)^{\alpha^{1}} \ldots\left(x^{k}\right)^{\alpha^{k}}$ and $|\alpha|=\alpha^{1}+\cdots+\alpha^{k}$ for $\alpha \in \mathbb{N}^{k}$, where $\mathbb{N}$ stands for the set of non-negative integers.

For $s \geq 1$ we denote by $Z_{s}$ the set of $\left(i_{1}, \ldots, i_{s}, \alpha\right) \in\{1, \ldots, k\}^{s} \times \mathbb{N}^{k}$ with the properties that $i_{1}<\cdots<i_{s}$, and either $|\alpha|<r$ or $|\alpha|=r$ and $i_{s}<\max \left\{l \in\{1, \ldots, k\}: \alpha^{l}>0\right\}$. Moreover, let $Z_{0}$ denote the set of $\alpha \in \mathbb{N}^{k}$ such that $|\alpha| \leq r$. In [2] it is proved that the map $I_{s}: C_{s} \mathbb{D}_{k}^{r} \rightarrow \mathbb{R}^{Z_{s}}$ given by $I_{s}(D)\left(i_{1}, \ldots, i_{s}, \alpha\right)=D\left(x^{i_{1}}, \ldots, x^{i_{s}}\right)\left(x^{\alpha}\right)$ for every $D \in C_{s} \mathbb{D}_{k}^{r}$ and every $\left(i_{1}, \ldots, i_{s}, \alpha\right) \in Z_{s}$ is an isomorphism of vector spaces for every non-negative integer $s$.

For $s \geq 1$ we denote by $W_{s}$ the set of $\left(i_{1}, \ldots, i_{s}, \alpha\right) \in\{1, \ldots, k\}^{s} \times \mathbb{N}^{k}$ such that $i_{1}<\cdots<i_{s},|\alpha| \leq r, \alpha \neq 0$ and $i_{s}<\max \left\{l \in\{1, \ldots, k\}: \alpha^{l}>0\right\}$. Moreover, let $W_{0}$ denote the set of $\alpha \in \mathbb{N}^{k}$ such that $|\alpha| \leq r$ and $\alpha \neq 0$. Of course, $W_{s} \subset Z_{s}$ for every non-negative integer $s$.

Suppose now that $s \geq 1$.

For $\left(i_{1}, \ldots, i_{s-1}, \beta\right) \in W_{s-1}$ we put $i_{s}=\max \left\{l \in\{1, \ldots, k\}: \beta^{l}>0\right\}$ and $\alpha=\beta-e_{i_{s}}$, where $e_{1}, \ldots, e_{k}$ is the standard basis of the module $\mathbb{Z}^{k}$. It is easily seen that $\left(i_{1}, \ldots, i_{s}, \alpha\right) \in Z_{s} \backslash W_{s}$. Writing $\varphi_{s}\left(i_{1}, \ldots, i_{s-1}, \beta\right)=$ $\left(i_{1}, \ldots, i_{s}, \alpha\right)$ we get $\varphi_{s}: W_{s-1} \rightarrow Z_{s} \backslash W_{s}$.

For $\left(i_{1}, \ldots, i_{s}, \alpha\right) \in Z_{s} \backslash W_{s}$ we put $\beta=\alpha+e_{i_{s}}$. It is easily seen that $\left(i_{1}, \ldots, i_{s-1}, \beta\right) \in W_{s-1}$. Writing $\psi_{s}\left(i_{1}, \ldots, i_{s}, \alpha\right)=\left(i_{1}, \ldots, i_{s-1}, \beta\right)$ we get $\psi_{s}: Z_{s} \backslash W_{s} \rightarrow W_{s-1}$.

LEMMA 2. $\psi_{s} \circ \varphi_{s}=\operatorname{id}_{W_{s-1}}$ and $\varphi_{s} \circ \psi_{s}=\operatorname{id}_{Z_{s} \backslash W_{s}}$.

Proof. The proof is immediate.

If $\left(i_{1}, \ldots, i_{s}\right) \in \mathbb{N}^{s}$ and $i_{u} \neq i_{v}$ for all $u, v \in\{1, \ldots, s\}$ such that $u \neq v$, then we will write $P\left(i_{1}, \ldots, i_{s}\right)=\left(i_{\sigma(1)}, \ldots, i_{\sigma(s)}\right)$ and $\operatorname{sgn}\left(i_{1}, \ldots, i_{s}\right)=$ $\operatorname{sgn} \sigma$, where $\sigma$ is the permutation of $\{1, \ldots, s\}$ such that $i_{\sigma(1)}<\cdots<i_{\sigma(s)}$.

Lemma 3. If $B \in \mathbb{R}^{Z_{s}}$, then $B \in I_{s}\left(\operatorname{ker} \partial_{s}\right)$ if and only if

$$
\begin{aligned}
& B\left(i_{1}, \ldots, i_{s}, \alpha\right)=-\frac{1}{\alpha^{i_{s}}+1} \sum_{\substack{j \in\left\{l \in\{1, \ldots, k\}: \alpha^{l}>0\right\} \\
j \neq i_{1}, \ldots, i_{s}}} \alpha^{j} \operatorname{sgn}\left(i_{1}, \ldots, i_{s-1}, j\right) \\
& \times B\left(P\left(i_{1}, \ldots, i_{s-1}, j\right), \alpha+e_{i_{s}}-e_{j}\right)
\end{aligned}
$$


for every $\left(i_{1}, \ldots, i_{s}, \alpha\right) \in Z_{s} \backslash W_{s}$. Furthermore, the right hand side of (19) contains only $B\left(i_{1}, \ldots, i_{s}, \alpha\right)$ such that $\left(i_{1}, \ldots, i_{s}, \alpha\right) \in W_{s}$.

Proof. Since every $D \in C_{s} \mathbb{D}_{k}^{r}$ is skew-symmetric, the first assertion of our lemma may be reformulated as follows: if $D \in C_{s} \mathbb{D}_{k}^{r}$, then $D \in \operatorname{ker} \partial_{s}$ if and only if

$$
\begin{aligned}
& D\left(x^{i_{1}}, \ldots, x^{i_{s}}\right)\left(x^{\alpha}\right) \\
& \quad=-\frac{1}{\alpha^{i_{s}}+1} \sum_{\substack{j \in\left\{l \in\{1, \ldots, k\}: \alpha^{l}>0\right\} \\
j \neq i_{s}}} \alpha^{j} D\left(x^{i_{1}}, \ldots, x^{i_{s-1}}, x^{j}\right)\left(x^{\alpha+e_{i_{s}}-e_{j}}\right)
\end{aligned}
$$

for every $\left(i_{1}, \ldots, i_{s}, \alpha\right) \in Z_{s} \backslash W_{s}$.

On account of the above-mentioned result of [2], if $D \in C_{s} \mathbb{D}_{k}^{r}$, then $D \in$ ker $\partial_{s}$ if and only if $\left(\partial_{s} D\right)\left(x^{i_{1}}, \ldots, x^{i_{s-1}}\right)\left(x^{\beta}\right)=0$ for every $\left(i_{1}, \ldots, i_{s-1}, \beta\right)$ $\in Z_{s-1}$. Applying (8) we can rewrite this as

$$
\sum_{j \in\left\{l \in\{1, \ldots, k\}: \beta^{l}>0\right\}} \beta^{j} D\left(x^{i_{1}}, \ldots, x^{i_{s-1}}, x^{j}\right)\left(x^{\beta-e_{j}}\right)=0 .
$$

But (20) is equivalent to $(21)$ with $\left(i_{1}, \ldots, i_{s-1}, \beta\right)=\psi_{s}\left(i_{1}, \ldots, i_{s}, \alpha\right)$, which means that (20) holds if and only if $(21)$ holds for $\left(i_{1}, \ldots, i_{s-1}, \beta\right) \in W_{s-1}$, because of Lemma 2. Consequently, the first assertion of our lemma will be proved as soon as we can show that $(20)$ implies $(21)$ with $\left(i_{1}, \ldots, i_{s-1}, \beta\right) \in$ $Z_{s-1} \backslash W_{s-1}$.

Since $Z_{0} \backslash W_{0}=\{0\}$, this is true if $s=1$. Therefore we can assume that $s \geq 2$. It is easy to see that for every $\left(i_{1}, \ldots, i_{s-1}, \beta\right) \in Z_{s-1} \backslash W_{s-1}$ and every $j \in\left\{l \in\{1, \ldots, k\}: \beta^{l}>0\right\} \backslash\left\{i_{1}, \ldots, i_{s-1}\right\}$, either $\beta-e_{j}=0$, or $\beta-e_{j} \neq 0$ and $\max \left\{i_{1}, \ldots, i_{s-1}, j\right\}=i_{s-1} \geq \max \left\{l \in\{1, \ldots, k\}:\left(\beta-e_{j}\right)^{l}>0\right\}$, and so $\left(P\left(i_{1}, \ldots, i_{s-1}, j\right), \beta-e_{j}\right) \in Z_{s} \backslash W_{s}$. Hence $(20)$ and the skew-symmetry of $D$ give

$$
\begin{aligned}
& D\left(x^{i_{1}}, \ldots, x^{i_{s-1}}, x^{j}\right)\left(x^{\beta-e_{j}}\right)=-\frac{1}{\beta^{i_{s-1}}+1} \\
& \times \sum_{\substack{h \in\left\{l \in\{1, \ldots, k\}: \beta^{l}>0\right\} \\
h \neq i_{1}, \ldots, i_{s-1}, j}} \beta^{h} D\left(x^{i_{1}}, \ldots, x^{i_{s-2}}, x^{h}, x^{j}\right)\left(x^{\beta+e_{i_{s-1}}-e_{h}-e_{j}}\right) .
\end{aligned}
$$

Substituting this into (21) and using the skew-symmetry of $D$ we see that the left hand side of (21) equals

$$
-\frac{1}{\beta^{i_{s-1}}+1} \sum_{\substack{j, h \in\left\{l \in\{1, \ldots, k\}: \beta^{l}>0\right\} \\ j, h \neq i_{1}, \ldots, i_{s-1} \\ j \neq h}} \beta^{h} \beta^{j} D\left(x^{i_{1}}, \ldots, x^{i_{s-2}}, x^{h}, x^{j}\right)\left(x^{\beta+e_{i_{s-1}}-e_{h}-e_{j}}\right),
$$


which vanishes because of the skew-symmetry of $D$. This proves the first assertion of our lemma.

To prove the second one it suffices to notice that for every $\left(i_{1}, \ldots, i_{s}, \alpha\right) \in$ $Z_{s} \backslash W_{s}$ and every $j \in\left\{l \in\{1, \ldots, k\}: \alpha^{l}>0\right\} \backslash\left\{i_{1}, \ldots, i_{s}\right\}$ we have $\max \left\{i_{1}, \ldots, i_{s-1}, j\right\}<i_{s}=\max \left\{l \in\{1, \ldots, k\}:\left(\alpha+e_{i_{s}}-e_{j}\right)^{l}>0\right\}$, and so $\left(P\left(i_{1}, \ldots, i_{s-1}, j\right), \alpha+e_{i_{s}}-e_{j}\right) \in W_{s}$. This completes the proof.

Note that Lemma 3 gives a description of ker $\partial_{s}$. Namely, any $B \in$ $I_{s}\left(\right.$ ker $\left.\partial_{s}\right)$ may be defined arbitrarily on $W_{s}$ first, and then by (19) on $Z_{s} \backslash W_{s}$ (the same is true for $s=0$ when the right hand side of (19) vanishes). In particular, we have the following corollary.

COROLlary. For every $s \geq 0$ the map ker $\left.\partial_{s} \ni D \mapsto I_{s}(D)\right|_{W_{s}} \in \mathbb{R}^{W_{s}}$ is an isomorphism of vector spaces.

Finally, from Lemmas 2 and 3 and the above-mentioned result of [2] we obtain the following theorem which states that the algebras $\mathbb{D}_{k}^{r}$ are acyclic.

TheOREM 3. $H_{s} \mathbb{D}_{k}^{r}=\{0\}$ for every $s \geq-1$.

Proof. dimker $\partial_{s-1}=\operatorname{card} W_{s-1}=\operatorname{card} Z_{s}-\operatorname{card} W_{s}=\operatorname{dim} C_{s} \mathbb{D}_{k}^{r}-$ $\operatorname{dim} \operatorname{ker} \partial_{s}=\operatorname{dimim} \partial_{s}$ for every $s \geq 1$.

\section{References}

[1] J. Dębecki, Linear liftings of p-forms to q-forms on Weil bundles, Monatsh. Math. 148 (2006), 101-117.

[2] - Linear natural liftings of forms to Weil bundles with Weil algebras $\mathbb{D}_{k}^{r}$, Note Mat., to appear.

[3] M. Doupovec and J. Kurek, Liftings of covariant (0,2)-tensor fields to the bundle of k-dimensional 1-velocities, Rend. Circ. Mat. Palermo (2) Suppl. 43 (1996), 111-121.

[4] D. J. Eck, Product-preserving functors on smooth manifolds, J. Pure Appl. Algebra 42 (1986), 133-140.

[5] J. Gancarzewicz and S. Mahi, Lifts of 1-forms to the tangent bundle of higher order, Czechoslovak Math. J. 40 (115) (1990), 397-407.

[6] J. Gancarzewicz, W. M. Mikulski and Z. Pogoda, Lifts of some tensor fields and connections to product preserving functors, Nagoya Math. J. 135 (1994), 1-41

[7] G. Kainz and P. W. Michor, Natural transformations in differential geometry, Czechoslovak Math. J. 37 (112) (1987), 584-607.

[8] I. Kolář, Jet-like approach to Weil bundles, seminar lecture notes, Masaryk Univ., Brno, 2001.

[9] I. Kolář, P. W. Michor and J. Slovák, Natural Operations in Differential Geometry, Springer, Berlin, 1993.

[10] O. O. Luciano, Categories of multiplicative functors and Weil's infinitely near points, Nagoya Math. J. 109 (1988), 69-89. 
[11] W. M. Mikulski, The natural operators lifting 1-forms on manifolds to the bundles of A-velocities, Monatsh. Math. 119 (1995), 63-77.

[12] - The geometrical constructions lifting tensor fields of type $(0,2)$ on manifolds to the bundles of A-velocities, Nagoya Math. J. 140 (1995), 117-137.

Instytut Matematyki

Uniwersytet Jagielloński

Łojasiewicza 6

30-348 Kraków, Poland

E-mail: debecki@im.uj.edu.pl

Received 18.9.2008

and in final form 28.10.2008 